\title{
Vibration analysis of carbon nanotubes-based zeptogram masses sensors and taking into account their rotatory inertia
}

\author{
A. Azrar $^{1}$, L. Azrar ${ }^{2,3}$, and A. A. Aljinaidi ${ }^{3}$ \\ ${ }^{1}$ MMC, Faculty of Sciences and Techniques of Tangier, Abdelmalek Essaâdi University; Tangier; Morocco. \\ ${ }^{2}$ Research Center STIS, Higher School of Technical Education of Rabat (ENSET), Mohammed V University in Rabat, \\ Morocco. \\ ${ }^{3}$ Department of Mechanical Engineering, Faculty of Engineering, Kind Abdulaziz University, Jeddah, Saudi Arabia
}

\begin{abstract}
In this research work, the transverse vibration behaviour of single-walled carbon nanotubes (SCNT) based mass sensors is studied using the Timoshenko beam and nonlocal elasticity theories. The nonlocal constitutive equations are used in the formulations and the CNT with different lengths, attached mass (viruses and bacteria) and the general boundary conditions are considered. The dimensionless frequencies and associated modes are obtained for one and two attached masses and different boundary conditions. The effects of transverse shear deformation and rotatory inertia, nonlocal parameter, length of the carbon nanotubes, and attached mass and its location are investigated in detail for each considered problem. The relationship between the frequencies and mode shapes of the sensor and the attached zeptogramme masses are obtained. The sensing devices for biological objects including viruses and bacteria can be elaborated based on the developed sensitivity and frequency shift methodological approach.
\end{abstract}

\section{Introduction}

Detection of the mass of bio-molecules has become an increasing growing field in the biological and biomedical sciences. It is recognized as one of the key technologies for predictive and preventive medicine [1]. The CNT is ultra light and is highly sensitive to its environment changes. Therefore, many researchers have explored the potential of using CNT as nano mechanical resonators in atomic-scale mass sensor [2,3]. Length scale effect analysis on vibration behavior of single walled carbon nanotubes with generalized boundary conditions and CNT conveying fluid have been elaborated by Azrar et al [4-7].

The aim of this paper is analyzing the dynamic behavior of a CNT-based biosensor where the rotary inertia of the attached bio-object and of the CNT is taken into account. The analytical solutions are obtained based on the on iterative-incremental procedures. The effect of the physical and geometrical parameters are investigated.

\section{Mathematical formulation}

Let us consider a slender single walled carbon nanotube of length L, diameter d, thickness h, moments of inertia I, cross section area A, the mass density $\rho$, Young modulus $\mathrm{E}$ and shear modulus $\mathrm{G}$. The nonlocal elasticity theory combined with the Timoshenko beam model is adopted. The dynamic governing equations are formulated.

\subsection{Dynamic equation of the SWCNT sensor based on nonlocal TBT}

Based on the nonlocal elasticity and Timoshenko beam theories (TBT), the governing equations of the nonlocal CNT can be obtained as [4-7]

$$
\left\{\begin{array}{l}
k s A G\left(\frac{\partial \boldsymbol{\theta}}{\partial x}+\frac{\partial^{2} \mathbf{w}}{\partial x^{2}}\right)-\rho A\left[1-\left(e_{0} a\right)^{2} \frac{\partial^{2}}{\partial x^{2}}\right] \frac{\partial^{2} \mathbf{w}}{\partial t^{2}}=0 \\
E I \frac{\partial^{2} \boldsymbol{\theta}}{\partial x^{2}}-k s A G\left(\theta+\frac{\partial \mathbf{w}}{\partial x}\right)-\rho I\left[1-\left(e_{0} a\right)^{2} \frac{\partial^{2}}{\partial x^{2}}\right] \frac{\partial^{2} \boldsymbol{\theta}}{\partial t^{2}}=0
\end{array}\right.
$$

where $\mathrm{w}$ denotes the displacement, $\boldsymbol{\theta}$ the rotation of the cross section and $\mathrm{e}_{0} \mathrm{a}$ is the nonlocal parameter.

For the free vibrations, harmonic motion is assumed $\mathbf{w}(x, t)=\mathbf{W}(x) e^{i \omega t} ; \quad \boldsymbol{\psi}(x, t)=\boldsymbol{\Psi}(x) e^{i \omega t}$ where $\omega$ is the natural vibration frequency parameter. For dimensionless equations, the following variables are used.

$y=\frac{x}{L} ; W=\frac{w}{L} ; \mu=\frac{e_{0} a}{L} ; \xi=\frac{E A L^{2}}{E I} ; \zeta=\frac{E I}{k_{s} A G L^{2}} ; \beta=\sqrt{\frac{\rho A L^{4}}{E I}} ;$ 


$$
\left\{\begin{array}{l}
\frac{d \boldsymbol{\Psi}}{d y}+\frac{d^{2} \mathbf{W}}{d y^{2}}+\beta^{2} \zeta\left[\mathbf{W}-\mu^{2} \frac{d^{2} \mathbf{W}}{d y^{2}}\right]=0 \\
\zeta\left(1-\mu^{2} \xi \beta^{2}\right) \frac{d^{2} \boldsymbol{\Psi}}{d y^{2}}-\frac{d \mathbf{W}}{d y}+\left(\zeta \xi \beta^{2}-1\right) \boldsymbol{\Psi}=0
\end{array}\right.
$$

Eliminating $\mathbf{W}$ or $\boldsymbol{\Psi}$ from eqs. (5) and (6) yields to the following uncoupled differential equations:

$$
\begin{aligned}
& \left(\mu^{2} \beta^{2}(\zeta+\xi)-\mu^{4} \zeta \xi \beta^{4}-1\right) \frac{d^{4} \mathbf{W}}{d y^{4}}+\left(2 \mu^{2} \zeta \xi \beta^{4}\right. \\
& \left.-\left(\mu^{2}+\zeta+\xi\right) \beta^{2}\right) \frac{d^{2} \mathbf{W}}{d y^{2}}+\left(1-\zeta \xi \beta^{2}\right) \beta^{2} \mathbf{W}=0 \\
& \left(\mu^{2} \beta^{2}(\zeta+\xi)-\mu^{4} \zeta \xi \beta^{4}-1\right) \frac{d^{4} \boldsymbol{\Psi}}{d y^{4}}+\left(2 \mu^{2} \zeta \xi \beta^{4}\right. \\
& \left.-\left(\mu^{2}+\zeta+\xi\right) \beta^{2}\right) \frac{d^{2} \boldsymbol{\Psi}}{d y^{2}}+\left(1-\zeta \xi \beta^{2}\right) \beta^{2} \boldsymbol{\Psi}=0
\end{aligned}
$$

The analytical solution of the ordinary differential equation Eq. (7) is sought in the following form

$\mathbf{W}(y)=\mathbf{A} e^{i \lambda y}$

where $A$ is a constant.

Substituting Eq. (9) in Eq.(7), the following characteristic equation is obtained:

$\left(\mu^{2} \xi \beta^{2}-1\right)\left(\mu^{2} \zeta \beta^{2}-1\right) \lambda^{4}+\left(\left(2 \zeta \xi \beta^{2}-1\right) \mu^{2}-\xi-\zeta\right) \beta^{2} \lambda^{2}$

$+\left(\zeta \xi \beta^{2}-1\right) \beta^{2}=0$

The solutions of this equation are given by:

$\lambda_{1}=\sqrt{\frac{\left(\left(1-2 \zeta \xi \beta^{2}\right) \mu^{2}+\xi+\zeta\right) \beta^{2}+\sqrt{\Delta}}{2}} ; \quad \lambda_{2}=-\lambda_{1} ;$

$\lambda_{3}=i \sqrt{\frac{\sqrt{\Delta}-\left(\left(1-2 \zeta \xi \beta^{2}\right) \mu^{2}+\xi+\zeta\right) \beta^{2}}{2}} ; \lambda_{4}=-\lambda_{3}$;

where $\Delta=\left(\mu^{4}+(\xi-\zeta)^{2}-2(\xi-\zeta) \mu^{2}\right) \beta^{4}+4 \beta^{2}$

The transverse displacement and rotation solutions of (5, 6) are given by:

$$
\begin{gathered}
\mathbf{W}_{1}=A_{1} e^{i \lambda_{1} y}+A_{2} e^{i \lambda_{2} y}+A_{3} e^{i \lambda_{3} y}+A_{4} e^{i \lambda_{4} y} ; 0 \leq y<y_{1} \\
\mathbf{W}_{2}=A_{5} e^{i \lambda_{1} y}+A_{6} e^{i \lambda_{2} y}+A_{7} e^{i \lambda_{3} y}+A_{8} e^{i \lambda_{4} y} ; y_{1} \leq y<y_{2} \\
\mathbf{W}_{3}=A_{9} e^{i \lambda_{1} y}+A_{10} e^{i \lambda_{2} y}+A_{11} e^{i \lambda_{3} y}+A_{12} e^{i \lambda_{4} y} ; y_{2} \leq y<1 \\
\mathbf{\Psi}_{i}=\frac{\left(\left(\mu^{2} \xi \beta^{2}-1\right) \zeta^{2} \beta^{2}-1\right)}{1-\zeta \xi \beta^{2}}\left(\frac{d \mathbf{W}_{i}}{d y}\right) \\
+\frac{\zeta\left(1-\mu^{2} \xi \beta^{2}\right)\left(\mu^{2} \zeta \beta^{2}-1\right)}{1-\zeta \xi \beta^{2}}\left(\frac{d^{3} \mathbf{W}_{i}}{d y^{3}}\right)
\end{gathered}
$$

where $A_{\mathrm{i}}$ are constants to be determined with the boundary conditions at $\mathrm{y}=0$ and 1 and the continuity equations at $\mathrm{y}_{1}$ and $\mathrm{y}_{2}$ while $\mathbf{W}_{1}, \mathbf{W}_{2}$ and $\mathbf{W}_{3}$ and $\boldsymbol{\Psi}_{1}, \boldsymbol{\Psi}_{2}$ and $\boldsymbol{\Psi}_{3}$ are, respectively, the left, central and right transverse displacements and rotations divided at the points where the concentrated masses are attached.

Introducing the following non-dimensional coordinates:

$$
y=x / L ; y_{1}=x_{1} / L_{1} ; y_{2}=x_{2} / L_{2}
$$

The boundary conditions are:

$$
\begin{array}{ll}
\mathbf{M}_{1}(0)-K_{r}{ }_{r} \frac{d \mathbf{W}_{1}(0)}{d y}=0 & \mathbf{M}_{3}(1)+K_{r}{ }^{R} \frac{d \mathbf{W}_{3}(1)}{d y}=0 \\
\mathbf{Q}_{1}(0)+K_{t}^{L} \mathbf{W}_{1}(0)=0 & \mathbf{Q}_{3}(1)-K_{t}{ }^{R} \mathbf{W}_{3}(1)=0
\end{array}
$$

where $K_{r}^{L}, K_{t}^{L}$ and $K_{r}^{R}, K_{t}^{R}$ are the translational and the rotational spring constants at the left and right ends of the $\mathrm{CNT}$ at $\mathrm{y}=0$ and $\mathrm{y}=1$, see "figure 1 '. The classical boundary conditions can be simply obtained as special cases when the stiffness's of the springs take some extreme values such as zero and infinity.

The continuity equations at the position $\mathrm{y}_{1}$ are:

$$
\begin{array}{ll}
\left.\mathbf{W}_{1}(y)\right|_{y=y_{1}}=\left.\mathbf{W}_{2}(y)\right|_{y=y_{1}} & (16-a) \\
\left.\frac{d \mathbf{W}_{1}}{d y}\right|_{y=y_{1}}=\left.\frac{d \mathbf{W}_{2}}{d y}\right|_{y=y_{1}} & (16-b) \\
\mathbf{M}_{1}+\left.c_{1}^{2} \bar{m}_{1} \beta^{4} \mathbf{W}_{1}\right|_{y=y_{1}}=\left.\mathbf{M}_{2}\right|_{y=y_{1}} & (16-c) \\
\mathbf{Q}_{1}+\left.\bar{m}_{1} \beta^{4} \mathbf{W}_{1}\right|_{y=y_{1}}=\left.\mathbf{Q}_{2}\right|_{y=y_{1}} & (16-d)
\end{array}
$$

and at $\mathrm{y}_{2}$

$$
\begin{aligned}
& \left.\mathbf{W}_{2}(y)\right|_{y=y_{2}}=\left.\mathbf{W}_{3}(y)\right|_{y=y_{2}} \\
& \left.\frac{d \mathbf{W}_{2}}{d y}\right|_{y=y_{2}}=\left.\frac{d \mathbf{W}_{3}}{d y}\right|_{y=y_{2}} \\
& \mathbf{M}_{2}+\left.c_{2}^{2} \bar{m}_{2} \beta^{4} \mathbf{W}_{2}\right|_{y=y_{2}}=\left.\mathbf{M}_{3}\right|_{y=y_{2}} \\
& \mathbf{Q}_{2}+\left.\bar{m}_{2} \beta^{4} \mathbf{W}_{2}\right|_{y=y_{2}}=\left.\mathbf{Q}_{3}\right|_{y=y_{2}}
\end{aligned}
$$

where

$$
\begin{aligned}
\mathbf{M}_{i}= & \beta^{2}\left(\mu^{2}\left(\zeta \xi \beta^{2}-1\right)-\zeta\right) \mathbf{W}_{i}(y) \\
& -\left(\mu^{2} \xi \beta^{2}-1\right)\left(\mu^{2} \zeta \beta^{2}+1\right) \frac{d^{2} \mathbf{W}_{i}(y)}{d y^{2}} \\
\mathbf{Q}_{i}= & \frac{\beta^{2}\left(\left(1-2 \zeta \xi \beta^{2}\right) \mu^{2}+\xi+\zeta\right)}{\zeta \xi \beta^{2}-1} \frac{d \mathbf{W}_{i}(y)}{d y} \\
& +\frac{\left(\mu^{2} \xi \beta^{2}-1\right)\left(\mu^{2} \zeta \beta^{2}-1\right)}{\zeta \xi \beta^{2}-1} \frac{d^{3} \mathbf{W}_{i}(y)}{d y^{3}} \\
K_{r}^{L}= & \frac{k_{r}^{L} L}{E I} ; K_{r}^{R}=\frac{k_{r}^{R} L}{E I} ; K_{t}^{L}=\frac{k_{t}^{L} L^{3}}{E I} ; K_{t}^{R}=\frac{k_{t}^{R} L^{3}}{E I} \\
\bar{m}_{i}= & \frac{m_{i}}{\rho A L} ; c_{i}=\frac{r_{i}}{L} ;
\end{aligned}
$$




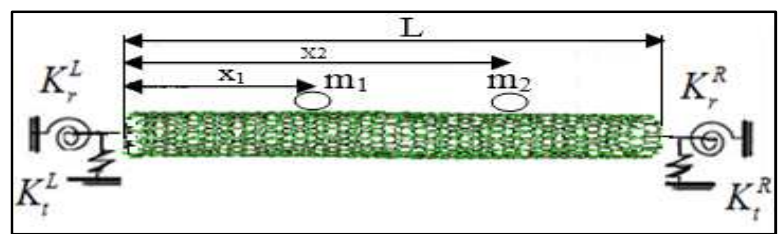

Fig. 1. Elastically restrained CNT at both ends.

\section{Frequency equation}

Substituting Eq. (12) into Eqs. (15)-(17), taking into account Eq. (14) one obtains the following system of equations expressed as

$\left[\begin{array}{rrrr}a_{1,1} & a_{1,2} & \mathrm{~L} & a_{1,12} \\ a_{2,1} & a_{2,2} & \mathrm{~L} & a_{2,12} \\ \mathrm{M} & \mathrm{M} & \mathrm{L} & \mathrm{M} \\ a_{12,1} & a_{12,2} & \mathrm{~L} & a_{12,12}\end{array}\right]\left[\begin{array}{c}A_{1} \\ A_{2} \\ \mathrm{M} \\ A_{12}\end{array}\right]=\left[\begin{array}{c}0 \\ 0 \\ \mathrm{M} \\ 0\end{array}\right]$

The non-triviality condition is established by solving:

$\operatorname{det}(\mathbf{H})=0$

where $\mathrm{H}$ is the matrix of the coefficients $\mathrm{a}_{\mathrm{i}, \mathrm{j}}$ of the system and the roots $\beta_{\mathrm{n}}$ are the eigenvalues of the problem. Eq.(18) is a hardly nonlinear function of $\beta_{n}$. A numerical technique based on the Newton-Raphson algorithm has been elaborated for numerical solutions.

\section{Numerical results and discussions}

Numerical results are presented using the following geometrical and material properties.

$\rho=2,3 e^{3} \mathrm{~kg} / \mathrm{m}^{3}, E=1 e^{3} G p a, G=420 G P a, v=0.19$,

$h=0.34 e^{-9} m, L=20 a, A=7.85 e^{-19} m^{2}, a=1.5 \times e^{-9} m$,

$d=10^{-9} \mathrm{~m}, I=\pi d^{4} / 64, k s=0.877, \Omega_{0}=E I / k s A G$,

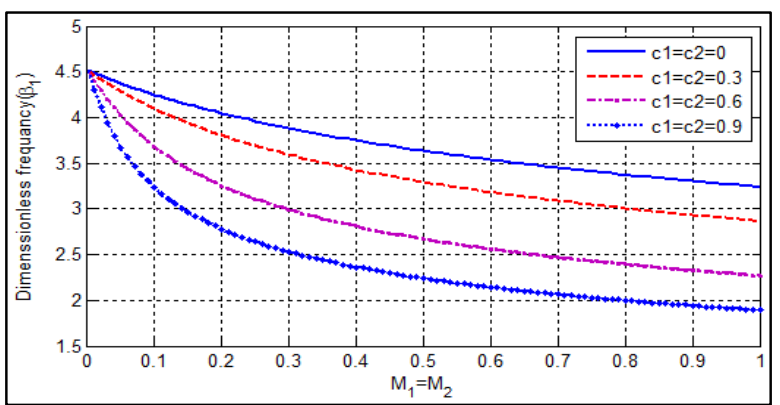

Fig. 2. Dimensionless frequencies as a function of dimensionless masses

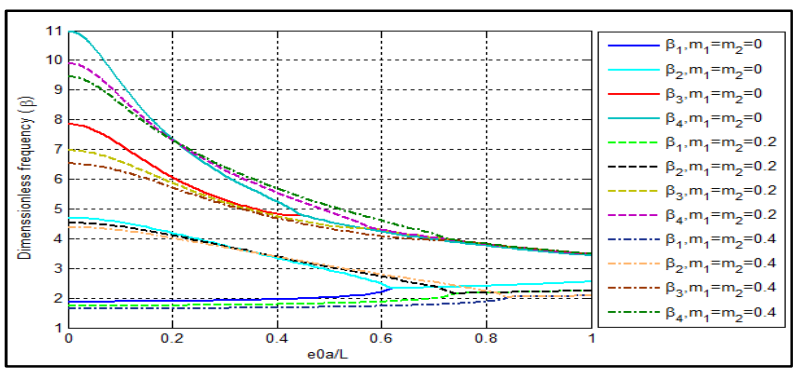

Fig. 3. Small length scale effect on the dimensionless frequency of a cantilever single walled CNT for different masses

Figure 2 shows the effect of mass inertia of the first dimensionless frequency of the clamped $K_{i}=+\infty$ sensor with attached mass. It can be seen that the frequency of the sensor decreases with increasing the attached mass and its inertia.

Figure 3 shows the variations of the eigenfrequency parameter associated to the first fourth modes shapes for a cantilever CNT with the nonlocal parameter $\mathrm{e}_{0} \mathrm{a} / \mathrm{L}$ for dimensionless masses. It is found that increasing the nonlocal parameter decreases the frequency and instability behaviors can be reached.

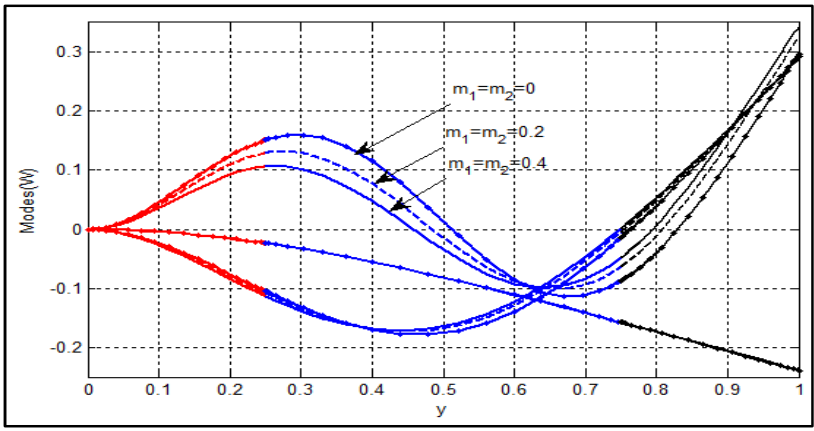

Fig. 4. The effect of zeptogram mass on the first three modes of cantilever SWCNT.

Figure 4 shows the effect of zeptogram mass on the first three modes of cantilever SWCNT. It can be seen that the attached mass has the most significant effect on mode shapes

\section{Acknowledgements}

The authors would like to acknowledge the technical and financial supports from the DSR and the King Abdulaziz University, Jeddah, Saudi Arabia.

\section{Conclusions}

The rotary inertia of the attached bio-object has a strong effect on the natural frequencies and cannot be simply neglected. The nonlocal Timoshenko beam model is more adequate than the nonlocal Euler- Bernoulli beam model for short CNT biosensors. The transverse frequency and mode shape distortion due to attached mass have been used as parameters for characterizing SWCNT as zeptogram mass sensors.

\section{REFERENCES}

[1] L. Hood, J.R. Heath, M.E. Phelps, B. Lin, Science 306, 640-643, (2004).

[2] W. Shi, Z.-B. Shen, X.-L. Peng, X.-F. Li, International Journal of Mechanical Sciences 115-116, 406-415, (2016)

[3] Z. B Shen, L. P. Sheng, X.F. Li, G. J. Tang, Physica E 44, 1169-1175, (2012). 
[4] A. Azrar, L. Azrar and A. A. Aljinaidi. Journal Mechanics of Advanced Materials and Structures, 23, 1245-1262, (2016).

[5] A. Azrar, L. Azrar and A. A. Aljinaidi. Journal Composite Structures, 125, 127-143, (2015).

[6] A. Azrar, L. Azrar and A. A. Aljinaidi. MATEC Web of Conferences 1, 09002, (2012).

[7] A. Azrar, L. Azrar and A. A. Aljinaidi, Revue de Mécanique Appliqué et Théorique, 2, 5, 475-484, (2011). 